\title{
The use of immunological tests in epidemiological work
}

\author{
HG ten DAM \& H SANSARRICQ \\ Division of Communicable Diseases, World Health Organization, \\ 1211 Geneva, Switzerland
}

Since some simple immunological tests are in an advanced stage of development in the field of leprosy, it is appropriate to consider their potential usefulness and utilization for epidemiological work. As has been shown in the field of tuberculosis, the development of an apparently simple test into a useful epidemiological tool requires close cooperation between immunologists and the epidemiologists, and there is little doubt that in this matter the experience gathered about the tuberculin test in the last 75 years can be most instructive.

A matter which may preoccupy both immunologists and clinicians is that a test for infection with leprosy could fail to give a positive result in individuals incubating lepromatous leprosy, as is assumed to be the case with the Mitsuda test. This possible shortcoming will not preclude its application for many epidemiological purposes. The long experience with the tuberculin test has led to a good insight into its quantitative aspects, and this experience can be readily transferred to any test aimed at detecting leprosy infection, be it a skin test or a simple serological test.

\section{Basic requirements}

Since in epidemiological work a test may have to be applied to large numbers of healthy people who, individually, may not directly benefit from the test, it must be acceptable to the population and absolutely harmless for ethical reasons. Any invasive method, such as a test requiring $20 \mathrm{ml}$ of venous blood, would have a very limited scope. On the other hand, a test like the tuberculin test, which is based on the intradermal injection of a minute amount of what is commonly called a "soluble antigen", would be acceptable in many programmes provided, of course, that the antigen is non-sensitizing or has no biological effect other than causing mild transitionary local induration and erythema. 
Fields of application

\section{EPIDEMIOLOGICAL STUDIES}

Longitudinal studies may be undertaken in which, for instance, the degree of sensitization, as expresised by the test, may be correlated with the risk of disease of different clinical types, or in which the occurrence of disease is studied in relation to the time of conversion to the test. Such studies will no doubt contribute to our understanding of the natural history of leprosy.

\section{SURVEILLANCE}

A test that reveals subclinical infection with $\mathrm{M}$. leprae would make it possible to determine the annual risk of infection in a population, which would be a most useful epidemiological index of the leprosy problem. If in leprosy, like in tuberculosis, subclinical infection is far more common than disease, its risk is more easily determined than the risk (or incidence) of disease, even more so because the work can be done in readily accessible, healthy population groups (school-age children). Unlike the incidence of disease which is a result of infection contracted at some time in the past, the risk of infection will reflect the current situation. Thus the test would be eminently suitable for epidemiological surveillance, which may be undertaken independently of the control programme. Epidemiological information of this kind is most useful in the planning of specific control measures.

\section{SCREENING}

Another field in which the test can be used most profitably is the selection of population groups for particular studies, such as vaccination trials. If the objective of the vaccination would be to confer protection in persons not yet infected, one would wish to eliminate or at least to identify the infected persons. In a controlled trial infected persons might contribute large numbers of cases to both the control and the vaccinated group, thus masking the efficacy of the vaccination to an unknown extent. Similarly, if the objective would be to reinforce the immunological defence in persons already infected, one would wish to limit the trial to such persons.

A screening test may be used most profitably in control programmes, in which it may identify persons at high risk or exclude persons who would not benefit from a particular health measure. 
It is reasonable to assume that, at least in uninfected persons, a vaccine would induce a measurable reaction to the test, and that for a particular vaccine the response would be dose-dependent. The test, therefore, may be used to evaluate vaccination programmes or different vaccination techniques and to compare qualitatively equal vaccines (batches). It will be most useful in vaccine development since it will allow many variables to be studied in small populations. For a new vaccine, for example, one would wish to study the dose-response curve, in terms of skin sensitivity and untoward reactions, to determine the still acceptable dose that gives the highest biological effect (in different age groups). It may also be necessary to study in man the keeping qualities of the vaccine, the method of administration, etc.

In BCG vaccination against tuberculosis the relation between tuberculin sensitivity and protection is not known, but the capacity of including sensitivity as a function of the acceptability of the vaccine (in terms of lesion size and complication rate) is nevertheless considered an important characteristic. The test, therefore, may be of a certain value in comparing qualitatively different vaccines.

\section{Shortcomings}

Whereas an ideal test would allow an infallible distinction to be made between persons with a certain condition and persons without, in practice any immunological test will be liable to experimental error. Part of this is systematic error inherent to the test, and determines whether the test result is a true reflection of the condition sought. For instance, during the first few weeks of infection children may not have developed skin sensitivity. If tested they will systematically be classified falsely as uninfected. This is a shortcoming that simply will have to be accepted. Sometimes it is possible to correct for this type of error, for instance, cases in far advanced stages of disease may no longer react to the test, but these are of course easily recognized as such.

Another part of experimental error may be called random error. It results from variations in the potency of the test substance, inaccuracies in applying the test, in observation and recording, etc. To a large extent the epidemiologist, unlike the clinician who applies a diagnostic test to a single individual, will be able to measure random error, and compensate for it, if necessary, by increasing the groups observed.

Being measurable, this type of error can be reduced to a minimum. The test substance used in a particular study, for instance, should be of uniform potency. Factors that could influence the potency, such as the effects of heat and light and adsorption to the container wall, should be investigated. Of primary importance in this con- 
nection is that the testing technique should be simple and standardized. Uniform, reliable equipment should be used. The testers and readers should receive thorough training, and if several testers and readers participate in the same study, their performances should be compared.

\section{Efficacy and effectiveness}

Any test will have a limited efficacy. It is of interest to note that this will depend both on its ability to detect persons with the sought condition among those actually having it and on its ability to detect persons without the sought condition among those free from it. These abilities, which are usually denoted as sensitivity and specificity, are of great importance if the test result has to be interpreted qualitatively (as "positive" or "negative"), for instance by a clinician who wishes to use the test in an individual case. The problem in such a case is how to select the criterion for what is positive and negative (and doubtful!). It arises because the effectiveness, or predictive value, or discriminative power (to mention only a few of the terms used) not only depends on sensitivity and specificity (as defined above) but also on extraneous circumstances. One of these is the prevalence of the sought condition in the population. If the condition is rare, one would wish to set the criterion high, so that the specificity of the test is high and overdiagnosis is avoided. The price to be paid for this is that the sensitivity is reduced, and thus that the risk of missing a true case is increased. If the condition is common the inverse applies.

In the case of the tuberculin test this problem used to be insignificant in European countries because doubtful intermediate reactions were very rare. In tropical countries, however, the population is often massively sensitized by atypical mycobacteria, as a result of which the specificity of the tuberculin test is highly reduced. (Probably in order to avoid blaming the test, the population was said to have "non-specific sensitivity".) As a diagnostic tool, therefore, the tuberculin test is quite useless in such countries. Yet it has been used effectively in a large number of epidemiological surveys. This was possible because in epidemiological work the criterion need not be set more or less arbitrarily, but can be determined according to the group results observed and moreover in accordance with the purpose of the test. Thus the epidemiologist will be able to set the criterion in sucii a way that he uses the test in the most effective way, as will be shown below.

Application and interpretation

\section{SURVEILLANCE}

To measure the prevalence of infection in a certain population group 
one would select a representative sample, apply the test to all individuals selected, and measure and record the reactions at the appropriate time. From the result obtained the frequency distribution of the reactions, by size, is constructed, and for visual inspection entered into a histogram.

If the test has been effective, there will be two distinct group effects: one group of "zero" reaction and only small ones, the other of reactions normally distributed around a higher mean, clearly distinct from the former (see Fig. 1). These will be the "negatives" and the "positives", respectively. Whatever these terms actually mean biologically, depends notably on the systematic error of the test; ideally they will correlate with absence or presence of infection, as can be verified by comparing the distribution with that in known patients (see Fig. 1). Owing to random error, there may be some overlapping of the two parts. The epidemiologist will divide this overlapping part of the distribution between the extremes in such a way that there will be as many false positives as false negatives. Even though all persons have not been classified correctly, an accurate estimate is obtained of the infection prevalence. By repeating the same procedure a few years later both the risk of infection and its trend can be calculated.

Fig. 1 DISTRIBUTION OF TUBERCULIN REACTION SIZES IN TUBERCULOSIS PATIENTS (TOP) AND IN A GENERAL VILLAGE POPULATION (BELOW)

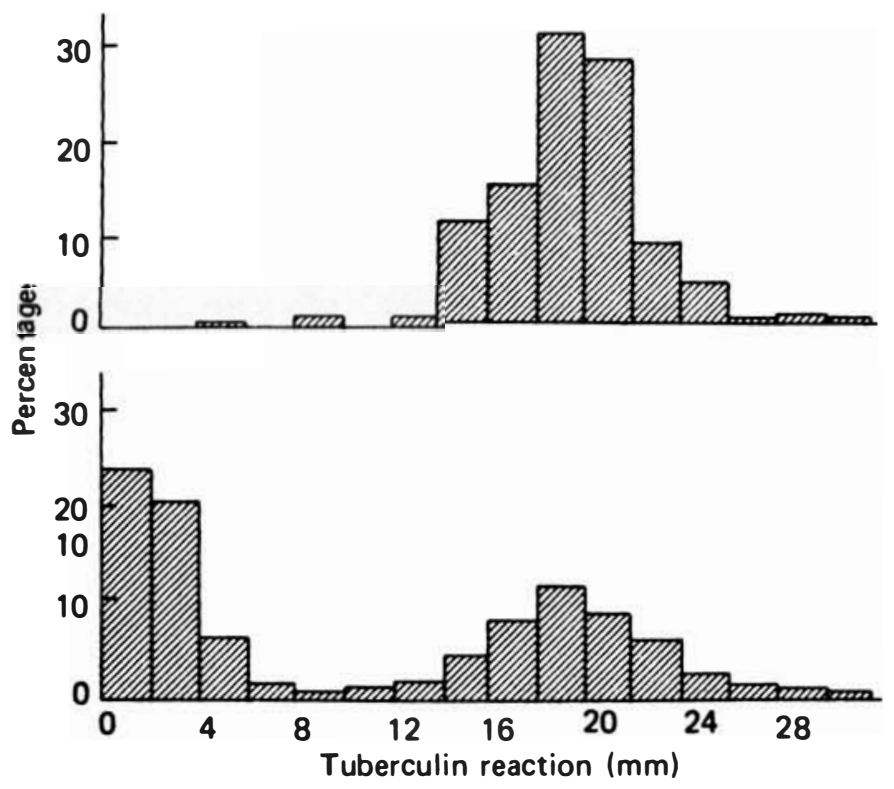

If the effectiveness of the test has been low, however, the effect will be as if there were a third group, of medium-sized reactions, overlapping the two. This will partially mask the distri- 
bution of "positive" reactions (see Fig. 2). The usual technique in this case is to reconstruct the left tail of the distribution of "positive" reactions from the right tail of the observed distribution. Thus a rough estimate of the infection prevalence can be made. By repeating the same procedure a few years later a similarly rough estimate of the infection prevalence at that time could be made. However, comparing more simply the right tails obtained on both occasions will give a fairly accurate estimate of the trend in the infection prevalence and the risk of infection, which is the main purpose of surveillance. What is done is that by setting the criterion high, the specificity of the test is increased to an acceptable level. The price paid for this is that the sensitivity is reduced and thus that the calculations can only be based on a fraction of the "positives" in the population examined.

Fig. 2 DISTRIBUTION OF TUBERCULIN REACTION SIZES IN UNVACCINATED CHILDREN, AGED 8.12 YEARS, IN A TROPICAL COUNTRY

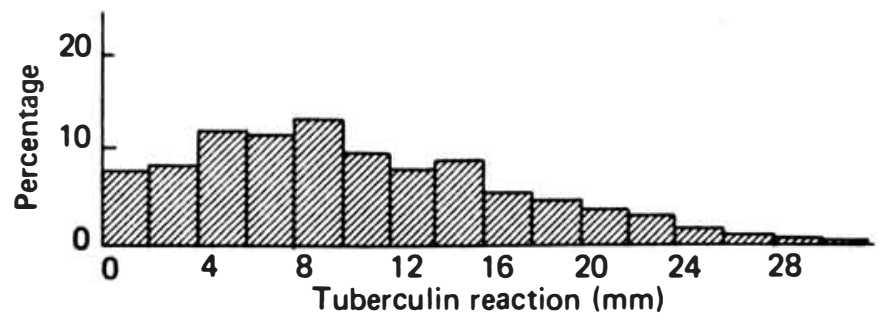

\section{SCREENING}

The actual method used in screening is largely the same as in prevalence surveys of infection. There are, however, two important differences, one favourable, one unfavourable.

To start with the unfavourable one, in screening, say for a vaccination trial, for practical reasons it is indicated to apply the vaccines and the placebo on the occasion that the test is read. Thus, the epidemiologist is deprived of the possibility of constructing his distributions to decide what is the best criterion. He will have to go by preliminary experience, and arbitrarily set a criterion to be applied in the trial, both in terms of the reaction sizes to be considered "negative" and "positive" and the test dose to be used. In the major BCG trials the limiting reaction size was 5 or $6 \mathrm{~mm}$, but dosages varied from 3 IU to 250 IU. This criterion, nevertheless, may be verified as soon as all the results of the screening have been collected. At that time persons can still be excluded according to the results obtained, if not entirely from the study at least from the analysis. On the other hand, persons excluded from the start obviously can no longer be included at this stage. For this reason the epidemiologist will set the preliminary criterion 
in such a way that most likely too many, rather than too few, persons are included. In this connection he will have to consider the cost of including some persons who would not contribute information. In the latest BCG trial in South India, all persons were vaccinated or given a placebo, irrespective of the tuberculin reaction (which was read after vaccination), but only persons with reactions up to $7 \mathrm{~mm}$ were considered in the evaluation of the protective effect.

The favourable circumstance is that in screening it is not necessary to divide the entire population into "negative" and "positive". It is merely required to identify the group with one or the other characteristic. Thus "doubtful" cases can simply be excluded. This means that, for instance, if a group of negative reactors were to be selected, the epidemiologist could set the test criterion relatively low. This certainly is indicated if the proportion of negative persons in the population is presumed to be high. The risk of including false negative reactors would be reduced at little cost. If the proportion of negative reactors were low, relatively many eligible persons tested might be lost to the study if the criterion were set too low. Yet, the need for setting a low criterion, to exclude false negative persons, would be greater. In this case the criterion would have to be set relatively high, and a careful adjustment made once the results of the screening were known. The fact that the epidemiologist can interpret the results of the screening test a posteriori, and analyse the trial accordingly is not always appreciated. The latest trial of BCG vaccination, in South India, has been criticized on the point that infected persons were included! The criticism is only justified for as far as one can maintain that the investigators could have been biased by the outcome of the trial when determing the criteria. The adjustment, therefore, should be made (and published) before the final results are known.

Incidentally, this procedure shows that often a preliminary study to determine which population (age) groups are best included in the trial may be very profitable, and reinforces the practical consideration that a trial is best undertaken in a population in which the proportion of eligible persons is high: in such a population the efficacy of the screening test is increased. Also, the need to record the results of the screening tests should be obvious. In the field of BCG vaccination against tuberculosis this has not always been realized.

In diagnostic screening for a control programme, such as mass vaccination or preventive treatment, the epidemiologist would handle the test criterion differently, and largely according to the cost involved. Thus, when screening for vaccination, he would probably include persons with doubtful reactions to a large extent because at least some of them would profit from this relatively inexpensive measure. On the other hand, for preventive treatment he would select initially only those persons with the highest risk of developing disease, and exclude doubtful cases. 


\section{EVALUATION}

Evaluation, for instance, of two batches of the same vaccine, or of administering a vaccine in two different ways, is a matter of comparison. In this respect, the test will have to be used strictly quantitatively. This means one has to measure the degree of skin sensitivity induced rather than its presence or absence. This point is often badly understood, and it is not unusual to hear investigators speak of "negative" and "positive" or of "conversion" after BCG vaccination. These expressions are biologically meaningless when applied to a homogeneous population (those vaccinated). Treatment of the data in this way is inefficient statistically. The post-vaccination reactions in the vaccinated usually will be normally distributed (see Fig. 3). Characterizing the distributions merely by the percentages of those "positive" gives much less information than providing the mean and the standard deviations of the reactions, especially since the criterion for "positive" would have to be set arbitrarily. All information about the "spread" of the reactions, and thus of the uniformity of the vaccination method, would be lost. Moreover, a badly chosen criterion may mask possible differences between batches, between techniques of administration, etc. Bias is easily introduced in this interpretation.

Fig. 3 DISTRIBUTION OF TUBERCULIN REACTION SIZES IN SCHOOLCHILDREN VACCINATED WITH DIFFERENT BATCHES OF BCG VACCINE

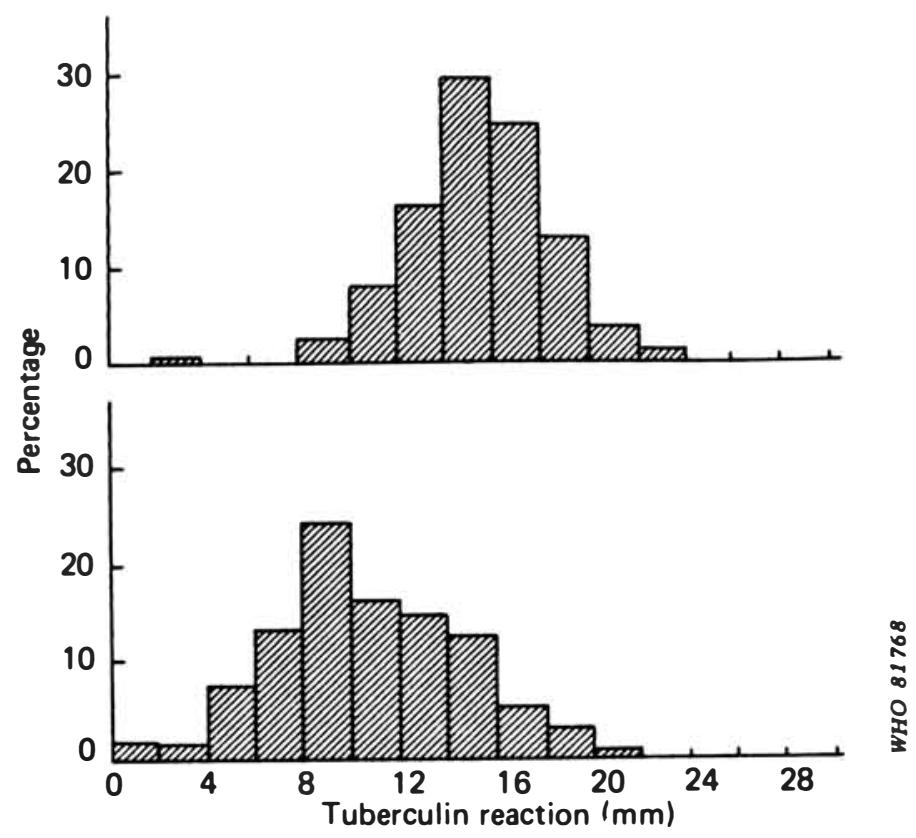


The correct method in an evaluation study, therefore, is to select a suitable population; to apply an appropriate screening test, as seen before; to apply randomly the different treatments to be studied; to apply at the optimal time the post-vaccination test; to present the distributions of the reactions obtained in the different treatment groups; and to determine the confidence interval of the difference of the means. Significant differences will be revealed, and, as the case may be biological equality can also be demonstrateed.

Experience as regards the standard deviation usually obtained with the test will make it possible to estimate the optimal group sizes for such studies.

\section{Validation}

A noticeable point.in the epidemiological use of a test, as described above, is that in each instance a measure is obtained of its effectiveness, systematic error apart. This implies that on the whole the epidemiologist will notice when the results obtained are inconclusive. Whereas it may be fortunate that such an event will be detected, it obviously should be avoided as far as possible. To achieve this, basic knowledge of the test should be available from some unambiguous studies. Partly this information should be provided by the immunologist who suggests the test. In general, several important variables can be studied in suitable animal models. These include preliminary data on the safety and the dose-response relationship, and information on variations in potency (keeping qualities). Other relevant data, however, should be directly obtained in studies in man. In this matter the epidemiologist can largely contribute. Thus, he could determine the optimal dosage and time of reading the reactions. Next, he could study the sensitivity and specificity, in populations in which the condition was known to be present or absent, and as regards the latter in which possibly interfering circumstances were known to exist ("non-specific" sensitivity) or could be introduced (vaccination). If alternative tests were available, he certainly would wish to compare those in various study populations. In these matters the services of an "independent" epidemiologist may well save many years of sterile discussions between immunologists.

Another aspect closely connected with the epidemiological use of a test is the relative unimportance of exact "standardization" or "calibration" of the test material. In the case of tuberculin, extensive studies in animals and man have been carried out to arrive at comparable products. These efforts were only partially successful since many products appeared to differ qualitatively to such an extent that if reactions in one population were similar, they differed in another. The objective of the exercise was partly to satisfy clinicians, who are in the unfortunate position of having to give a "standard" interpretation, and partly to achieve comparability in time and place of various surveys carried out. In an attempt to solve the problem, a batch of tuberculin was prepared sufficiently 
large for worldwide use for some 50 - 100 years. It soon appeared that this ultimum in standardization did not allow a "standard" interpretation to be given to the reactions observed in different surveys, because of the large experimental error from other factors including differences in reactivity between ethnic groups and systematic differences between readers. As has been seen, the epidemiologist simply interprets the results from the distribution observed. In view of this, slight variations in potency are of no consequence, provided, of course, that the same product is used in each study, and extensive calibration in man is not required for epidemiological work. For any particular product, calibration in an animal model will suffice.

\section{Conclusion}

A simple immunologiçal test could be profitably utilized in a variety of epidemiological work in leprosy. The epidemiologist will be able to handle almost any test, even if it may seem to lack sensitivity and specificity. In this respect the viewpoint of clinicians might be quite different.

Progress in this field will probably be achieved more rapidly if experienced epidemiologists are given the opportunity to participate in the development of the test. The relevant activities of the Scientific Working Group on Immunology of Leprosy (IMMLEP) are being developed according to this requirement. 